\title{
Double-Exponential Fast Gauss Transform Algorithms for Pricing Discrete Lookback Options
}

\author{
By \\ Yusaku Yaмамото*
}

\begin{abstract}
This paper presents fast and accurate algorithms for computing the prices of discretely sampled lookback options. Under the Black-Scholes framework, the pricing of a discrete lookback option can be reduced to a series of convolutions of a function with the Gaussian distribution. Using this fact, an efficient algorithm, which computes these convolutions by a combination of the double-exponential integration formula and the fast Gauss transform, has been proposed recently. We extend this algorithm to lookback options under Merton's jump-diffusion model and American lookback options. Numerical experiments show that our method is much faster and more accurate than conventional methods for lookback options under Merton's model. For American lookback options, our method outperforms conventional methods when required accuracy is relatively high.
\end{abstract}

\section{$\S 1 . \quad$ Introduction}

A lookback option is the right to sell an asset at the end of a time period at the highest price the asset took during the period (lookback put option), or to buy an asset at the lowest price it took during the period (lookback call option). It is one of the most popular exotic options and various types of lookback options are traded in the market. Usually, the maximum or minimum is taken over a finite set of time points within the period called monitoring dates, and such options are called discrete lookback options.

Communicated by H. Okamoto. Received November 24, 2004. Revised March 14, 2005. 2000 Mathematics Subject Classification(s): 65R10, 91B28, 62P05

Key words: option pricing, lookback options, fast Gauss transform, double-exponential formula

* Department of Computational Science and Engineering, Nagoya University, Nagoya 4648603, Japan. 
The price of a lookback option is given as the discounted expectation value of the option payoff under the risk-neutral probability measure [9]. In the case of discrete lookback options, it is difficult to find an explicit formula for the expectation value and many numerical methods have been proposed so far, including the Monte Carlo method, the binomial method [13][14][3] [2] and Reiner's convolution method [21]. The binomial method approximates the geometric Brownian motion of the underlying asset by a discrete process on a lattice. Reiner's convolution method, on the other hand, exploits the fact that the expectation value can be computed by a series of convolutions of a function with the transition probability density function (tpdf) of the asset price, and computes these convolutions with the fast Fourier transform.

Recently, Broadie and Yamamoto [8] proposed a fast and accurate algorithm for pricing discrete path-dependent options including the lookback option. Their method is also based on the representation of the expectation value by a series of convolutions and computes these convolutions efficiently by a combination of the double-exponential numerical integration formula [22] and the fast Gauss transform [11][12][16]. Numerical experiments show that their method is much faster and more accurate than conventional methods. However, their algorithm has been limited to the standard lookback options under the Black-Scholes model.

In this paper, we generalize their algorithm in two ways. First, we extend the algorithm to deal with lookback options under Merton's lognormal jumpdiffusion model [19]. This model is important because it can capture the heavy tails of the tpdf of the asset price, which are often observed in the market but cannot be described by the Black-Scholes model. Second, we propose a modified version of the algorithm that can price American lookback options, a variant of the lookback option with an early exercise feature. We compare our algorithms with the conventional pricing methods such as the Monte Carlo method, the binomial method and Reiner's convolution method and show the effectiveness of our approach.

This paper is organized as follows. In section 2, we formulate the pricing problem of lookback options and show how the pricing computation can be reduced to a series of convolutions. Sections 3 and 4 provide the details of our algorithms for the standard lookback options under Merton's jump-diffusion model and the American lookback options, respectively. Numerical results that illustrate the performance of our algorithms are shown in section 5. Finally, we give some concluding remarks in section 6 . 


\section{§2. Problem Formulation}

In this section, we formulate the pricing problem of lookback put options following [8]. The pricing of lookback call options can be done in a completely parallel manner, so we concentrate on put options in the following. Let the time period be $[0, T]$ and the monitoring dates be $t_{i}=i \Delta t(i=0,1, \ldots, n)$, where $\Delta t=T / n$. Also, denote the asset price at $t_{i}$ by $S_{i}$ and let

$$
M_{i}=\max _{1 \leq k \leq i} S_{k}
$$

Then the payoff of the lookback put option can be written as $M_{n}-S_{n}$ and its price at time 0 is given by

$$
V_{0}^{L P}\left(S_{0}\right)=e^{-r T} E_{0}\left[M_{n}-S_{n}\right],
$$

where $r$ is the riskless interest rate and $E_{t}[\cdot]$ is the conditional expectation value operator under the risk-neutral probability measure $Q$ given information up to time $t[9]$.

Eq. (2.2) involves two random variables $S_{n}$ and $M_{n}$ at time $T$, so we need the joint distribution function of them to compute the expectation value. To reduce the dimensionality of the problem, we apply a change of measure (see Babbs [2] and Andreasen [1]) and rewrite eq. (2.2) as:

$$
V_{0}^{L P}\left(S_{0}\right)=e^{-q T} S_{0} E_{0}^{\prime}\left[\frac{M_{n}}{S_{n}}-1\right],
$$

where $q$ is the dividend rate and $E_{t}^{\prime}[\cdot]$ denotes the conditional expectation operator under a new measure $Q^{\prime}$ defined by

$$
d Q^{\prime}=\frac{S_{n}}{S_{t} e^{(r-q)(T-t)}} d Q .
$$

By further introducing the log stock prices $s_{i}=\ln \left(S_{i} / S_{0}\right)$ and $m_{i}=\ln \left(M_{i} / S_{0}\right)$, we can finally write the option price as

$$
V_{0}^{L P}\left(S_{0}\right)=e^{-q T} S_{0} E_{0}^{\prime}\left[e^{m_{n}-s_{n}}-1\right] .
$$

This shows that it is sufficient to find the distribution of $m_{n}-s_{n}$ to compute the option price.

To compute eq. (2.5), we consider the distribution of $m_{i}-s_{i}(i=0,1, \ldots$, $n)$. Apparently, the distribution function is zero when $m_{i}-s_{i}<0$ and there is a finite probability mass at $m_{i}-s_{i}=0$. We therefore represent the distribution of $m_{i}-s_{i}$ by two quantities, namely, a scalar $c_{i}$ which represents the probability 
that $m_{i}-s_{i}=0$ and a function $g_{i}(x)(x>0)$ which represents the probability density in the region $m_{i}-s_{i}>0$. Note that the probability density function (pdf) of $m_{i}-s_{i}$ can be formally written as $c_{i} \delta(x)+g_{i}(x)$, where $\delta(x)$ is the Dirac delta function. At time 0, we have

$$
\begin{aligned}
c_{0} & =1 \quad \text { and } \\
g_{0}(x) & =0
\end{aligned}
$$

by definition. To compute $c_{i}$ and $g_{i}(x)$ given $c_{i-1}$ and $g_{i-1}(x)$, we use the identity:

$$
\begin{aligned}
m_{i}-s_{i} & =\max \left(0, m_{i-1}-s_{i}\right) \\
& =\max \left(0,\left(m_{i-1}-s_{i-1}\right)+\left(s_{i-1}-s_{i}\right)\right) .
\end{aligned}
$$

If the asset price follows a Levy process, as in the case of the Black-Scholes model or Merton's jump-diffusion model, the increment $s_{i-1}-s_{i}$ is independent of $m_{i-1}-s_{i-1}$ and eq. (2.8) suggests that the pdf of $m_{i}-s_{i}$ is obtained by computing the convolution of the pdf of $m_{i-1}-s_{i-1}$ with that of $s_{i-1}-s_{i}$ and collecting all the probability mass corresponding to $m_{i}-s_{i}<0$ to the point $m_{i}-s_{i}=0$. In summary, we have the following recursion formula:

$$
\begin{aligned}
\bar{g}_{i}(x) & =\int_{-\infty}^{\infty}\left\{c_{i-1} \delta(y)+g_{i-1}(y)\right\} f(x-y) d y \\
& =c_{i-1} f(x)+\int_{0}^{\infty} g_{i-1}(y) f(x-y) d y, \\
c_{i} & =\int_{-\infty}^{0} \bar{g}_{i}(x), \\
g_{i}(x) & =\bar{g}_{i}(x) \quad(x>0),
\end{aligned}
$$

where $f(x)$ is the probability density function of $s_{i-1}-s_{i}$. Equations (2.6), (2.7), (2.9), (2.10) and (2.11), along with eq. (2.5), provides us with a means of computing the lookback option price by a series of convolutions.

\section{§3. The DE-FGT Algorithm for Pricing Lookback Options under Merton's Jump-diffusion Model}

The model. Now we consider the case where the asset price follows the lognormal jump-diffusion model introduced by Merton [19]. Under the probability measure $Q$, the dynamics of $S_{i}$ is governed by the following equation:

$$
d S_{t} / S_{t-}=(r-q-\nu \lambda) d t+\sigma d W_{t}+d\left(\sum_{l=1}^{N_{0}^{P}(t)}\left(J_{l}-1\right)\right)
$$


where $W_{t}$ is a Wiener process, $N_{t}^{P}(s)$ is the number of jumps between $t$ and $t+s$, which follows a Poisson process with intensity $\lambda,\left\{J_{l}\right\}$ is a sequence of i.i.d. random variables whose distribution is given by

$$
\begin{gathered}
\ln \left(1+J_{l}\right) \sim N\left(\gamma-\frac{1}{2} \delta^{2}, \delta^{2}\right), \\
\nu=e^{\gamma}-1, \quad \text { and } \\
S_{t-}=\lim _{s \rightarrow t-0} S_{s} .
\end{gathered}
$$

The constants $\gamma$ and $\delta$ determine the mean and the standard deviation of the jumps, respectively. The processes $W_{t}, N_{t}^{P}(s)$ and $\left\{J_{l}\right\}$ are assumed to be mutually independent. This model is important because it introduces discontinuous changes in the asset price and thereby reproduces the heavy tails of the transition probability function, a characteristic which is often observed in the market but cannot be described by the Black-Scholes model.

In this model, the market becomes incomplete because of the existence of jumps and the standard option pricing argument based on the replicating portfolio [4] is no longer applicable. However, under the assumption that the jump risk is diversifiable, Merton [19] shows that the price can again be written as the discounted expectation value of the option payoff. Thus, the incompleteness of the market causes little problem from the computational point of view.

Computing the lookback option price by the recursion formula. To compute the lookback option price using the recursion formula (2.9), (2.10) and (2.11), we need to know the pdf of $s_{i-1}-s_{i}$ under the new probability measure $Q^{\prime}$ defined by eq. (2.4). Andreasen [1] shows that under $Q^{\prime}$, the asset price $S_{t}$ follows a new equation:

$$
d S_{t} / S_{t-}=\left(r-q-\nu \lambda+\sigma^{2}\right) d t+\sigma d W_{t}^{\prime}+d\left(\sum_{l=1}^{N^{\prime}{ }_{0}(t)}\left(J_{l}^{\prime}-1\right)\right)
$$

where $W_{t}^{\prime}$ is a Wiener process under $Q^{\prime},\left\{J_{l}^{\prime}\right\}$ is a sequence of i.i.d. random variables whose distribution is given by

$$
\ln \left(1+J_{l}^{\prime}\right) \sim N\left(\gamma+\frac{1}{2} \delta^{2}, \delta^{2}\right),
$$

and $N_{t}^{\prime}(s)$ is a Poisson process under $Q^{\prime}$ with modified intensity

$$
\lambda^{\prime}=\lambda(1+\nu)=\lambda e^{\gamma} .
$$


Andreasen's proof is based on Girsanov's theorem [15][20] for jump-diffusion processes. Alternatively, we can show by elementary arguments that an expectation value of the form:

$$
V_{0}\left(S_{0}\right)=e^{-r T} E_{0}\left[h\left(S_{0}, S_{1}, \ldots, S_{n}\right) S_{n}\right]
$$

can be computed as

$$
V_{0}\left(S_{0}\right)=e^{-q T} S_{0} E_{0}^{\prime}\left[h\left(S_{0}, S_{1}, \ldots, S_{n}\right)\right],
$$

where $E_{0}^{\prime}[\cdot]$ denotes the expectation value obtained by assuming that $S_{t}$ follows a modified process specified by eqs. (3.5), (3.6) and (3.7). See the appendix for the proof. Because $M_{n}$ is a function of $\left\{S_{0}, S_{1}, \ldots, S_{n}\right\}$, we can readily apply this fact to obtain eq. (2.3) from eq. (2.2).

By integrating eq. (3.5), we have

$$
S_{i}=S_{i-1} \exp \left\{\left(r-q+\frac{1}{2} \sigma^{2}-\nu \lambda\right) \Delta t+\sigma \sqrt{\Delta t} z_{0}^{\prime}+\sum_{l=1}^{N_{t}^{\prime P}(\Delta t)}\left(\delta z_{l}^{\prime}+\gamma+\frac{1}{2} \delta^{2}\right)\right\},
$$

where $z_{l}(l=0,1, \ldots)$ are independent random variables that follow the standard normal distribution $N(0,1)$ under $Q^{\prime}$. Thus we know that the pdf of $s_{i-1}-s_{i}$ has the following form:

$$
f(x)=\sum_{l=0}^{\infty} e^{-\lambda^{\prime} \Delta t} \frac{\left(\lambda^{\prime} \Delta t\right)^{l}}{l !} f^{(l)}(x)
$$

where

$$
\begin{aligned}
f^{(l)}(x) & =\frac{1}{\sqrt{2 \pi} \sigma_{l}} \exp \left\{-\frac{\left(x+\mu_{l}^{\prime}\right)^{2}}{2 \sigma_{l}^{2}}\right\}, \\
\mu_{l}^{\prime} & =\left(r-q+\frac{1}{2} \sigma^{2}-\nu \lambda\right) \Delta t+l\left(\gamma+\frac{1}{2} \delta^{2}\right), \quad \text { and } \\
\sigma_{l}^{2} & =\sigma^{2} \Delta t+l \delta^{2} .
\end{aligned}
$$

By truncating the infinite sum in eq. (3.11) at some $l_{\max }$ and inserting the result into eq. (2.9), we can express the integral in the recursive formula (2.9) as a sum of convolutions of $g_{i-1}(x)$ with Gaussian functions:

$$
\begin{aligned}
I_{i}(x) & \equiv \int_{0}^{\infty} g_{i-1}(y) f(x-y) d y \\
& \simeq \sum_{l=0}^{l_{\max }} e^{-\lambda^{\prime} \Delta t} \frac{\left(\lambda^{\prime} \Delta t\right)^{l}}{l !} \int_{0}^{\infty} g_{i-1}(y) f^{(l)}(x-y) d y .
\end{aligned}
$$


Application of the DE-FGT algorithm. Now that the convolution in eq. (2.9) have been decomposed into convolutions of a function with Gaussian distribution, we can apply the Double-Exponential Fast Gauss Transform algorithm proposed in [8]. In particular, we introduce a double-exponential type change of variables:

$$
y=\ln \left\{1+\exp \left(\frac{\pi}{2}\left(1+u-e^{-u}\right)\right)\right\}
$$

and apply it to each term of eq. (3.15), obtaining

$$
\begin{aligned}
I_{i}^{(l)}(x)= & \int_{0}^{\infty} g_{i-1}(y)^{(l)} f(x-y) d y \\
= & \int_{-\infty}^{\infty} g_{i-1}\left(\ln \left\{1+\exp \left(\frac{\pi}{2}\left(1+u-e^{-u}\right)\right)\right\}\right) \\
& \times f^{(l)}\left(x-\ln \left\{1+\exp \left(\frac{\pi}{2}\left(1+u-e^{-u}\right)\right)\right\}\right) \\
& \times \frac{\exp \left(\frac{\pi}{2}\left(1+u-e^{-u}\right)\right) \frac{\pi}{2}\left(1+e^{-u}\right)}{1+\exp \left(\frac{\pi}{2}\left(1+u-e^{-u}\right)\right)} d u .
\end{aligned}
$$

Discretizing the integral (3.17) with the trapezoidal rule with step size $h$ and truncating the infinite sum at $N^{-}$and $N^{+}$gives

$$
I_{i}^{(l)}\left(a_{j}\right) \simeq \sum_{k=N^{-}}^{N^{+}} g_{i-1}\left(a_{k}\right) f^{(l)}\left(a_{j}-a_{k}\right) w_{k} \quad\left(j=N^{-}, \ldots, N^{+}\right),
$$

where

$$
\begin{aligned}
a_{k} & =\ln \left\{1+\exp \left(\frac{\pi}{2}\left(1+k h-e^{-k h}\right)\right)\right\} \quad \text { and } \\
w_{k} & =h \frac{\exp \left(\frac{\pi}{2}\left(1+k h-e^{-k h}\right)\right) \frac{\pi}{2}\left(1+e^{-k h}\right)}{1+\exp \left(\frac{\pi}{2}\left(1+k h-e^{-k h}\right)\right)} .
\end{aligned}
$$

Here we chose to evaluate the integral at $\left\{a_{j}\right\}_{j=N^{-}}^{N^{+}}$because eq. (2.9) is used recursively and $g_{i}(x)$ computed from $I_{i}^{(l)}(x)$ is used as the integrand in the next iteration.

Eq. (3.18) has the form of discrete convolution of a sequence $\left\{g_{i-1}\left(a_{k}\right)\right\}$ with the Gaussian distribution. The direct evaluation of this convolution would require $O\left(N^{2}\right)$ work, where $N \equiv N^{+}-N^{-}+1$ is the number of sample points at each time step. However, we can reduce the work to $O(N)$ by using the fast Gauss transform [11][12][16], which expands the Gaussian kernel in eq. (3.18) with the Hermite functions [11] or with the Fourier series [12][16] and computes the convolution efficiently by allowing a predetermined error level. Thus we have established the DE-FGT algorithm for lookback options under Merton's model. 
Computational work. Since the computation of eq. (2.9) requires $l_{\max }+1$ fast Gauss transforms and the recursion occurs $n$ times, the total work for computing the price of a lookback option is $O\left(n N l_{\max }\right)$ (excluding the small amount of work needed to compute eqs. (2.10) and (2.5)). However, Greengard and Strain [11] suggest an extension of the fast Gauss transform which can compute the convolution of a function with sum of multiple Gaussians (or Hermite functions) in only $O(N)$ work. Using this technique, the total computational work can be reduced to $O(n N)$. This is smaller than the $O(n N \log N)$ work needed by Reiner's method [21], which has been known as the fastest method so far. In addition, when $N$ increases, the error of the double exponential formula is expected to decrease faster than the error of the Simpson's rule used in Reiner's method. Thus we can expect that our method has an advantage over Reiner's method.

A note on the form of the change of variables. It would be appropriate here to make some comments on the form of the change of variables defined by eq. (3.16). As can be easily seen, eq. (3.16) approaches the doubleexponential transform $y=\exp \left(\frac{\pi}{2} \exp (-u)\right)$ when $u \rightarrow-\infty$ and the linear transform $y=\frac{\pi}{2} u$ when $u \rightarrow \infty$. We chose this form because the integrand decays like Gaussian when $y \rightarrow \infty$. In fact, this choice has proved effective in the pricing of various types of path-dependent options including the knock-out options, hindsight options and Bermudan options [8]. However, other types of double-exponential transforms are also applicable and it would be interesting to compare the performance of different transforms.

\section{§4. The DE-FGT Algorithm for Pricing American Lookback Options}

Pricing by dynamic programming. In this section, we consider the pricing of American lookback options, for which exercise prior to the maturity $T$ is permitted. More precisely, we treat a variant for which early exercise is possible only at discrete time points in $[0, T]$ called exercise dates (This type of options are sometimes referred to as Bermudan lookback options). For simplicity, the exercise dates are assumed to coincide with the monitoring dates, though it is easy to remove this restriction. As the asset price dynamics, we assume the Black-Scholes model or Merton's jump-diffusion model.

It is well known that the rational price of an American lookback option is given by the following expression:

$$
V_{0}^{L P}\left(S_{0}\right)=\sup _{\iota} E_{0}\left[e^{-r t_{\iota}}\left(M_{\iota}-S_{\iota}\right)\right]
$$


where $\iota$ denotes Markov stopping time [9]. That is, we consider expectation values of the option payoff under all possible stopping times (i.e. exercise strategies) and take the supremum. To find this supremum, we can use dynamic programming and compute the option value backward in time using the recursion [9]:

$$
V_{i}^{L P}\left(S_{i}, M_{i}\right)=\max \left(M_{i}-S_{i}, e^{-r \Delta t} E_{i}\left[V_{i+1}^{L P}\right]\right) .
$$

Here, $V_{i}^{L P}$ is the option value at time $t_{i}$ and is a function of $M_{i}$ and $S_{i}$ in general and $E_{i}[\cdot]$ is the expectation value operator given information up to $t_{i}$. $M_{i}-S_{i}$ is the profit obtained from exercising the right immediately at time $t_{i}$ (the exercise value), while $e^{-\Delta t} E_{i}\left[V_{i+1}^{L P}\right]$ is the discounted expectation value of the option value at the next exercise date (the continuation value). Eq. (4.2) states that it is optimal to exercise the option at time $t_{i}$ if the exercise value is greater than the continuation value, and to hold the option otherwise. The initial condition for the recursion (4.2) is given by

$$
V_{n}^{L P}\left(S_{n}, M_{n}\right)=M_{n}-S_{n} .
$$

Reduction to a 1-dimensional problem. The main computational task in the pricing of American lookback option is the evaluation of the expectation value in eq. (4.2), which involves two random variables $S_{i+1}$ and $M_{i+1}$. To reduce the dimensionality of the problem, we introduce a new variable $V_{i}^{L P}=V_{i}^{L P} / S_{i}$ and rewrite the backward recursion formula as follows:

$$
V_{i}^{L P}\left(S_{i}, M_{i}\right)=\max \left(M_{i} / S_{i}-1, e^{-r \Delta t} E_{i}\left[V_{i+1}^{L P}\right] / S_{i}\right) .
$$

By introducing a new measure $Q^{\prime}$ defined by

$$
d Q^{\prime}=\frac{S_{i+1}}{S_{t} e^{(r-q)\left(t_{i+1}-t\right)}} d Q,
$$

we have

$$
e^{-r \Delta t} E_{i}\left[V_{i+1}^{L P}\right]=S_{i} e^{-q \Delta t} E_{i}^{\prime}\left[V_{i+1}^{L P} / S_{i+1}\right],
$$

where $E_{i}^{\prime}[\cdot]$ is the expectation value operator under $Q^{\prime}$. Inserting this into eq. (4.4) gives

$$
V_{i}^{L P}\left(S_{i}, M_{i}\right)=\max \left(M_{i} / S_{i}-1, e^{-q \Delta t} E_{i}^{\prime}\left[V_{i+1}^{\prime L P}\right]\right) .
$$

Furthermore, it can be shown by induction that $V_{i}^{L P}\left(S_{i}, M_{i}\right)$ is a function of $M_{i} / S_{i}=e^{m_{i}-s_{i}}$ only. Denoting this function by $\tilde{V}_{i}^{L P}$, we finally obtain

$$
\tilde{V}_{i}^{L P}\left(e^{m_{i}-s_{i}}\right)=\max \left(e^{m_{i}-s_{i}}-1, e^{-q \Delta t} E_{i}^{\prime}\left[\tilde{V}_{i+1}^{L P}\right]\right) .
$$


Thus we have reached a 1-dimensional problem, for which the only variable is $m_{i}-s_{i}$.

Computation of the expectation value. To compute the expectation value $E_{i}^{\prime}\left[\tilde{V}_{i+1}^{L P}\right]$, we need to know the transition probability density function $p\left(m_{i+1}-s_{i+1} \mid m_{i}-s_{i}\right)$. Since

$$
\left.m_{i+1}-s_{i+1}=\max \left(0,\left(m_{i}-s_{i}\right)\right)+\left(s_{i}-s_{i+1}\right)\right)
$$

(See eq. (2.8)), we know that $m_{i+1}-s_{i+1}$ is obtained by adding a random variable $s_{i}-s_{i+1}$ to $m_{i}-s_{i}$ and resetting the result to zero if it is negative. Hence

$$
p(x \mid y)=f(x-y) h(x)+\delta(x) \int_{-\infty}^{0} f(x-y) d x,
$$

where $f(x)$ is the probability density function of $s_{i}-s_{i+1}$ (as defined in section 2) and $h(x)$ is the Heaviside step function which is 1 when $x \geq 0$ and 0 otherwise. Thus the expectation value when $m_{i}-s_{i}=y$ can be computed as

$$
\begin{aligned}
E_{i}^{\prime}\left[\tilde{V}_{i+1}^{L P}\right] & =\int_{-\infty}^{\infty} p(x \mid y) \tilde{V}_{i+1}^{L P}\left(e^{x}\right) d x \\
& =\int_{-\infty}^{0} f(x-y) d x \cdot \tilde{V}_{i+1}^{L P}\left(e^{0}\right)+\int_{0}^{\infty} f(x-y) \tilde{V}_{i+1}^{L P}\left(e^{x}\right) d x
\end{aligned}
$$

Eqs. (4.8) and (4.11), along with the initial condition

$$
\tilde{V}_{n}^{L P}\left(e^{m_{n}-s_{n}}\right)=e^{m_{n}-s_{n}}-1,
$$

which follows from eq. (4.3) enable us to compute the option price at time 0 using backward recursion.

Application of the DE-FGT algorithm. Now that we have expressed the option price by a series of convolutions of a function with (a sum of) Gaussian distribution, we can apply our DE-FGT algorithm. However, notice that the function $\tilde{V}_{i+1}^{(L P)}$, which appears as the integrand in eq. (4.11), is defined using the max operator (see eq. (4.8)) and therefore has discontinuity in its first and higher order derivatives. So straightforward application of the DE formula to eq. (4.11) would not result in the fast convergence rate characteristic of the formula.

To solve this problem, at each time step, we first use the bisection method to find the value $b$ of $m_{i}-s_{i}$ at which the exercise value and the continuation 
value are equal, divide the integration interval in the second term of eq. (4.11) into two subintervals $[0, b]$ and $[b, \infty)$, and apply the DE formula to each of the subinterval. Thus the integrand is smooth in each subinterval and the integration error is expected to decrease rapidly with the number of sample points. Note that this technique cannot be used with Reiner's method, because it uses a fixed step size $h$ for all time steps (a restriction imposed by the use of FFT), but the length of the subinterval $[0, b]$ is not a multiple of $h$ in general.

The computational work of the DE-FGT algorithm for American lookback options is $O(n N)$ when the number of exercise dates is $n$ and the number of sample points at each date is $N$.

\section{$\S 5 . \quad$ Numerical Results}

We implemented our DE-FGT algorithms for lookback options and compared its speed and accuracy with that of the conventional methods such as the Monte Carlo method, the binomial method and Reiner's convolution method [21]. All the numerical experiments were done on a $2.0 \mathrm{GHz}$ Pentium IV PC with Red-Hat Linux and GNU C++ compiler.

\section{§5.1. Lookback options under Merton's model}

We show the results for discrete lookback put options under Merton's model in Figures 1 and 2. The parameters are $S_{0}=100, r=0.1, q=0$, $\sigma=0.3, \lambda=2.0, \delta=0.3, \gamma=0$, and $T=0.2$. The number of monitoring dates, $n$, is 25 for Figure 1 and 50 for Figure 2. These frequencies correspond to bi-daily monitoring and daily monitoring, respectively. As the reference prices against which to compute the errors, we used $V_{0}^{L P}=12.09911864$ for $n=25$ and $V_{0}^{L P}=12.57499666$ for $n=50$, both of which were computed by Reiner's method with 256,000 sample points. We expect these values to be correct to at least ten digits after the decimal point because Reiner's method is a convergent method and the results for 64,000, 128,000 and 256,000 sample points agreed within the error of $10^{-10}$.

In the figures, the vertical axis and the horizontal axis represent the error in the calculated option price and the computation time, respectively, both in $\log$ scale. In the computation of eq. (3.17), we truncated the integral at the lower bound $u_{\min }=-4.0$ and the upper bound $u_{\max }=20 \sigma \sqrt{T}$ and used $N$ sample points to approximate the integral. The values of $N$ are shown in the graph. $l_{\max }$ in eq. (3.15) was set to 20. For the fast Gauss 


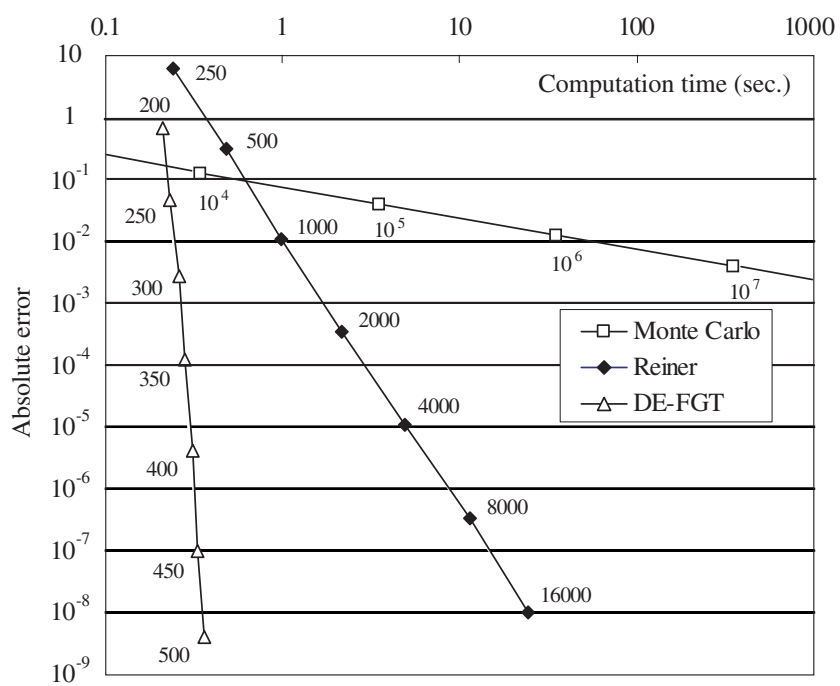

Figure 1. Computation time and accuracy of the three algorithms for pricing discrete lookback options under Merton's model (25 monitoring dates).

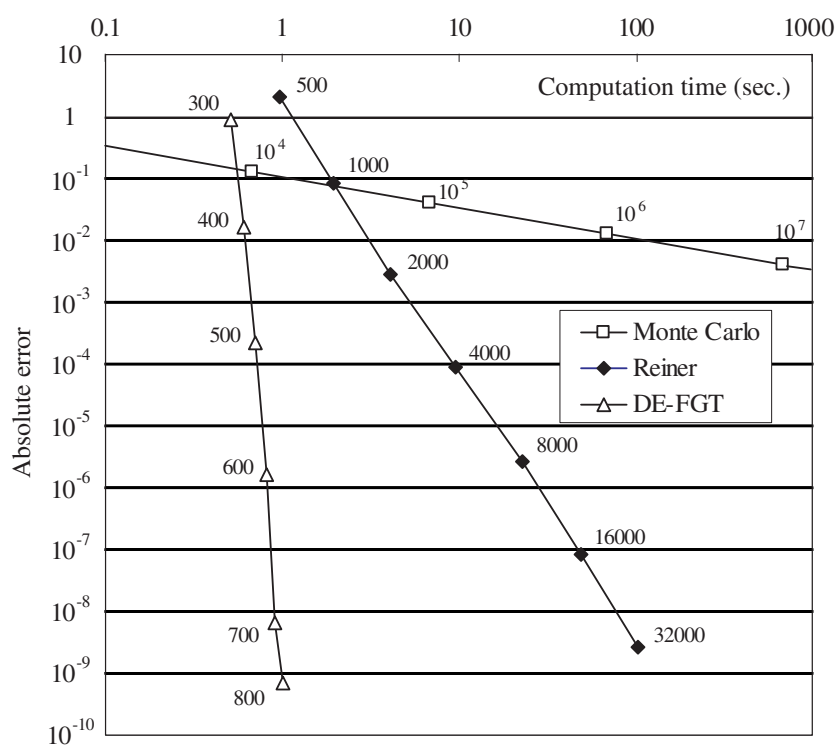

Figure 2. Computation time and accuracy of the three algorithms for pricing discrete lookback options under Merton's model (50 monitoring dates). 
transform, we used Greengard and Strain's algorithm [11]. This algorithm was also used for the American lookback options to be described in the next subsection. For comparison, we also plotted the computation time and accuracy for the Monte Carlo method and Reiner's method. The number of sample paths for the Monte Carlo method and the number of sample points for Reiner's method are also shown in the graphs. We did not try the binomial method because it takes into account only transitions to adjacent lattice points and is therefore not appropriate for pricing options under jump-diffusion models.

It is clear from the graphs that our DE-FGT algorithm converges much faster than the conventional Monte Carlo method or Reiner's convolution method and can compute the option prices within 1.0 second to an accuracy of $10^{-9}$.

\section{§5.2. American lookback options}

Results for the American lookback options under the Black-Scholes model are shown in Figures 3 and 4 . The parameters are $S_{0}=100, r=0.1, q=0$, $\sigma=0.3$ and $T=0.2$. The number of exercise dates, $n$, is 5 for Figure 3 and 10 for Figure 4. The reference prices computed by Reiner's method with 409,600 sample points are $V_{0}^{L P}=7.05538954$ for $n=5$ and $V_{0}^{L P}=7.92740313$ for $n=10$. These values are expected to be correct to at least nine digits after the decimal point because the results for 102,400, 204, 800 and 409, 600 sample points agreed within the error of $10^{-9}$.

In this case, we compared three methods, namely, our DE-FGT method, the binomial method [13] and Reiner's convolution method. We didn't try the Monte Carlo method because, as is well known, the standard MC method cannot deal with early exercise features. Although several attempts have been made to overcome this difficulty [5][6][17], these methods are basically for options depending on multiple assets and are inherently slower than the binomial method for options on a single asset. In the graphs, the number of sample points for the DE-FGT method and Reiner's method and the number of time steps for the binomial method are shown.

The results show that Reiner's method is the fastest when required accuracy is relatively low. Still, our method is competitive when higher accuracy is needed. Also, the convergence of Reiner's method is rather irregular. This seems to be because of the discontinuity in the first derivative of the integrand function, which we mentioned at the end of the previous section. In contrast, our method exhibits much smoother convergence behavior. 


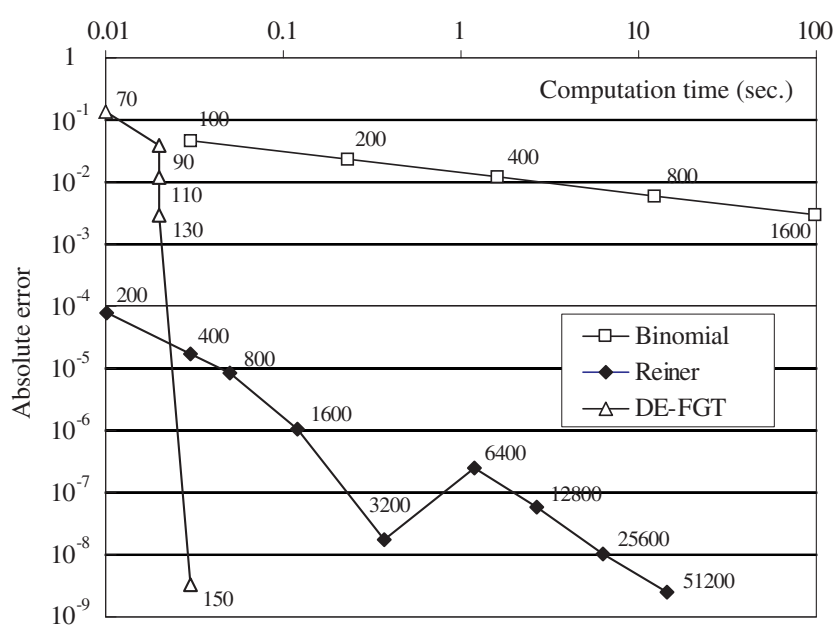

Figure 3. Computation time and accuracy of the three algorithms for pricing American lookback options under the BS model (5 exercise dates).

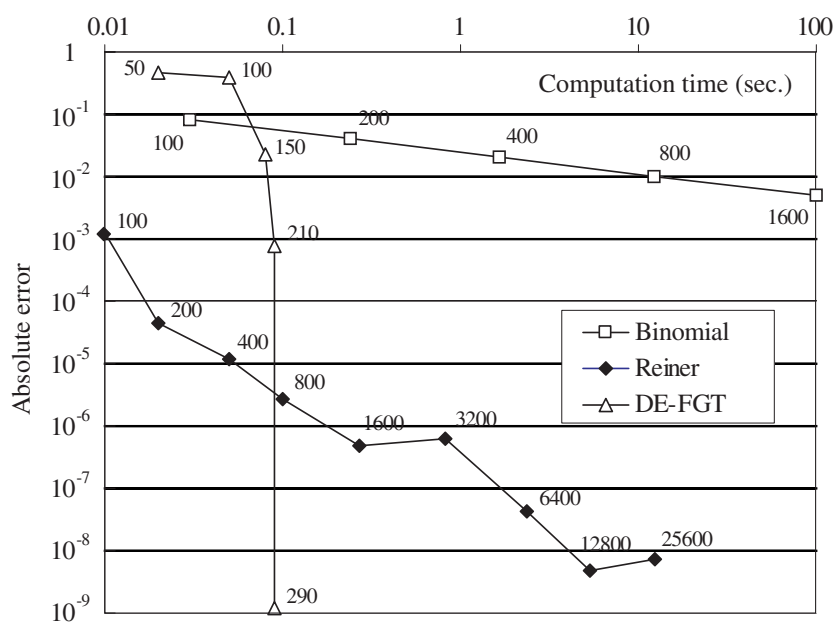

Figure 4. Computation time and accuracy of the three algorithms for pricing American lookback options under the BS model (10 exercise dates). 


\section{$\S 6 . \quad$ Conclusion}

In this paper, we proposed new pricing algorithms based on the doubleexponential integration formula and the fast Gauss transform for lookback options under Merton's jump-diffusion model and American lookback options. For lookback options under Merton's model, our method outperforms conventional methods such as Reiner's convolution method and can compute the option price within 1 second up to an accuracy of $10^{-9}$. For American lookback options, Reiner's method is the fastest when required accuracy is relatively low. But our method is competitive when higher accuracy is required. In addition, the convergence of our method is much smoother.

Future work includes extension of our algorithms to more general jumpdiffusion asset price models such as the variance gamma models and stochastic volatility models with jumps [10], and extension to other types of exotic options such as options on two or more assets and various types of path-dependent options.

\section{Acknowledgement}

I would like to thank Professor Mark Broadie for fruitful discussion. I am also grateful to Professor Masaaki Sugihara and Professor Hisashi Okamoto for providing me with the opportunity to present this study at the workshop "Thirty Years of the Double Exponential Transforms" held at Research Institute for Mathematical Sciences, Kyoto University. I'm indebted to the anonymous referee whose comment helped me to improve the paper. This work was partially supported by the Ministry of Education, Science, Sports and Culture, Grant-in-Aid for Young Scientists, 16760053, 2004 and Grant-in-Aid for the 21st Century COE "Frontiers of Computational Science".

\section{Appendix}

Theorem 6.1. Suppose that $S_{t}$ follows the stochastic differential equation (3.1) and we want to compute the expectation value

$$
V_{0}\left(S_{0}\right)=e^{-r T} E_{0}\left[h\left(S_{0}, S_{1}, \ldots, S_{n}\right) S_{n}\right] .
$$

$V_{0}$ can be computed as

$$
V_{0}\left(S_{0}\right)=e^{-q T} S_{0} E_{0}^{\prime}\left[h\left(S_{0}, S_{1}, \ldots, S_{n}\right)\right],
$$

where $E_{0}^{\prime}[\cdot]$ is the expectation value obtained by assuming that $S_{t}$ follows a modified process specified by eqs. (3.5), (3.6) and (3.7). 
Proof. By integrating eq. (3.1) from $t_{i}$ to $t_{i+1}$, we obtain the relationship between $S_{i}$ and $S_{i+1}$ as follows:

$$
S_{i}=S_{i-1} \exp \left\{\left(r-q-\frac{1}{2} \sigma^{2}-\nu \lambda\right) \Delta t+\sigma \sqrt{\Delta t} z_{0}+\sum_{l=1}^{N_{t}^{P}(\Delta t)}\left(\delta z_{l}+\gamma-\frac{1}{2} \delta^{2}\right)\right\},
$$

where $z_{l}(l=0,1, \ldots)$ are independent random variables that follow the standard normal distribution $N(0,1)$ under $Q$. Let

$$
x_{i} \equiv \ln \left(\frac{S_{i}}{S_{i-1}}\right) \quad(i=1,2, \ldots, n) .
$$

Then $x_{i}$ 's are mutually independent and follow the same distribution whose pdf is:

$$
p\left(x_{i}\right)=\sum_{l=0}^{\infty} e^{-\lambda \Delta t} \frac{(\lambda \Delta t)^{l}}{l !} p^{(l)}\left(x_{i}\right)
$$

where

$$
\begin{aligned}
p^{(l)}\left(x_{i}\right) & =\frac{1}{\sqrt{2 \pi} \sigma_{l}} \exp \left\{-\frac{\left(x_{i}-\mu_{l}\right)^{2}}{2 \sigma_{l}^{2}}\right\} \\
\mu_{l} & =\left(r-q-\frac{1}{2} \sigma^{2}-\nu \lambda\right) \Delta t+l\left(\gamma-\frac{1}{2} \delta^{2}\right), \quad \text { and } \\
\sigma_{l}^{2} & =\sigma^{2} \Delta t+l \delta^{2}
\end{aligned}
$$

Using $x_{i}$, eq. (6.1) can be rewritten as

$$
\begin{aligned}
V_{0}\left(S_{0}\right)= & e^{-r T} \int_{-\infty}^{\infty} d x_{1} \cdots \int_{-\infty}^{\infty} d x_{n} h\left(S_{0}, S_{0} e^{x_{1}}, \ldots, S_{0} e^{x_{1}+\cdots+x_{n}}\right) S_{0} e^{x_{1}+\cdots+x_{n}} \\
& \times p\left(x_{1}\right) \cdots p\left(x_{n}\right) \\
= & e^{-r T} S_{0} \int_{-\infty}^{\infty} d x_{1} \cdots \int_{-\infty}^{\infty} d x_{n} h\left(S_{0}, S_{0} e^{x_{1}}, \ldots, S_{0} e^{x_{1}+\cdots+x_{n}}\right) \\
& \quad \times p\left(x_{1}\right) e^{x_{1}} \cdots p\left(x_{n}\right) e^{x_{n}} .
\end{aligned}
$$


From eq. (6.5) through eq. (6.8) we have

$$
\begin{aligned}
p\left(x_{i}\right) e^{x_{i}} & \sum_{l=0}^{\infty} e^{-\lambda \Delta t} \frac{(\lambda \Delta t)^{l}}{l !} \frac{1}{\sqrt{2 \pi} \sigma_{l}} \exp \left\{-\frac{\left(x_{i}-\mu_{l}\right)^{2}}{2 \sigma_{l}^{2}}\right\} e^{x_{i}} \\
= & \sum_{l=0}^{\infty} e^{-\lambda \Delta t} \frac{(\lambda \Delta t)^{l}}{l !} \frac{1}{\sqrt{2 \pi} \sigma_{l}} \exp \left\{-\frac{\left(x_{i}-\left(\mu_{l}+\sigma_{l}^{2}\right)\right)^{2}}{2 \sigma_{l}^{2}}\right\} \exp \left\{\mu_{l}+\frac{1}{2} \sigma_{l}^{2}\right\} \\
= & \sum_{l=0}^{\infty} e^{-\lambda \Delta t} \frac{(\lambda \Delta t)^{l}}{l !} \frac{1}{\sqrt{2 \pi} \sigma_{l}} \exp \left\{-\frac{\left(x_{i}-\left(\mu_{l}+\sigma_{l}^{2}\right)\right)^{2}}{2 \sigma_{l}^{2}}\right\} \\
= & e^{(r-q) \Delta t} \sum_{l=0}^{\infty} e^{-\lambda(1+\nu) \Delta t} \frac{(\lambda(1+\nu) \Delta t)^{l}}{l !} \frac{1}{\sqrt{2 \pi} \sigma_{l}} \exp \left\{-\frac{\left(x_{i}-\left(\mu_{l}+\sigma_{l}^{2}\right)\right)^{2}}{2 \sigma_{l}^{2}}\right\} \\
= & e^{(r-q) \Delta t} \sum_{l=0}^{\infty} e^{-\lambda^{\prime} \Delta t} \frac{\left(\lambda^{\prime} \Delta t\right)^{l}}{l !} \frac{1}{\sqrt{2 \pi} \sigma_{l}} \exp \left\{-\frac{\left(x_{i}-\mu_{l}^{\prime}\right)^{2}}{2 \sigma_{l}^{2}}\right\},
\end{aligned}
$$

where we used eqs. (3.3), (3.7) and (3.13). If we define a new function

$$
\tilde{p}\left(x_{i}\right)=\sum_{l=0}^{\infty} e^{-\lambda^{\prime} \Delta t} \frac{\left(\lambda^{\prime} \Delta t\right)^{l}}{l !} \frac{1}{\sqrt{2 \pi} \sigma_{l}} \exp \left\{-\frac{\left(x_{i}-\mu_{l}^{\prime}\right)^{2}}{2 \sigma_{l}^{2}}\right\}
$$

we can rewrite eq. (6.9) as

$$
\begin{aligned}
V_{0}\left(S_{0}\right)=e^{-q T} & S_{0} \int_{-\infty}^{\infty} d x_{1} \cdots \int_{-\infty}^{\infty} d x_{n} h\left(S_{0}, S_{0} e^{x_{1}}, \ldots, S_{0} e^{x_{1}+\cdots+x_{n}}\right) \\
& \times \tilde{p}\left(x_{1}\right) \cdots \tilde{p}\left(x_{n}\right)
\end{aligned}
$$

Noting that $\tilde{p}\left(x_{i}\right)$ is the probability distribution function of $x_{i}$ under the assumption that $S_{t}$ follows a modified process specified by eqs. (3.5), (3.6) and (3.7), we can write the right hand side of eq. (6.12) as

$$
e^{-q T} S_{0} E_{0}^{\prime}\left[h\left(S_{0}, S_{1}, \ldots, S_{n}\right)\right] .
$$

This completes the proof. 


\section{References}

[1] Andreasen, J., The pricing of discretely sampled Asian and lookback options: a change of numeraire approach, J. Comput. Finance, 2 (1998), 5-30.

[2] Babbs, S., Binomial valuation of lookback options, J. Econom. Dynam. Control, 24 (2000), 1499-1525.

[3] Barraquand, J. and Pudet, T., Pricing of American path-dependent contingent claims, Math. Finance, 6 (1996), 17-51.

[4] Black, F. and Scholes, M., The pricing of options and corporate liabilities, J. Polit. Econ., 81 (1973), 637-654.

[5] Broadie, M. and Glasserman, P., Pricing American-style securities using simulation, J. Econom. Dynam. Control, 21 (1997), 1323-1352.

[6] A stochastic mesh method for pricing high-dimensional American options, $J$. Comput. Finance, 7 (2004), 35-72.

[7] Broadie, M. and Yamamoto, Y., Application of the fast Gauss transform to option pricing, Management Science, 49 (2003), 1071-1088.

[8] , A double-exponential fast Gauss transform algorithm for pricing discrete pathdependent options, to appear in Oper. Res.

[9] Duffie, D., Dynamic Asset Pricing Theory, 3rd ed., Princeton University Press, 1996.

[10] Duffie, D., Pan, J. and Singleton, K., Transform analysis and asset pricing for affine jump diffusions, Econom., 68 (2000), 1343-1376.

[11] Greengard, L. and Strain, J., The fast Gauss transform, SIAM J. Sci. and Statistical Computing, 12 (1991), 79-94.

[12] Greengard, L. and Sun, X., A new version of the fast gauss transform, Doc. Math. Extra Volume ICM 1998, III (1998), 575-584.

[13] Hull, J., Options, Futures and Other Derivatives, 5th ed., Prentice-Hall, 2002.

[14] Hull, J. and White, A., Efficient procedures for valuing European and American pathdependent options, Journal of Derivatives, 1 (1993), 21-31.

[15] Karatzas, I. and Shreve, S., Brownian Motion and Stochastic Calculus, 2nd ed., Springer-Verlag, 1997.

[16] Kobayashi, K., A remark on the fast Gauss transform, Publ. RIMS, Kyoto Univ., 39 (2003), 785-796.

[17] Longstaff, F. and Schwartz, E., Valuing American options using simulation: a simple least-squares approach, Rev. Financial Stud., 14 (2001), 113-147.

[18] Madan, D., Carr, P. and Chang, E., The variance gamma process and option pricing, European Finance Review, 2 (1988), 79-105.

[19] Merton, R., Continuous-Time Finance, Basil Blackwell, 1992.

[20] Mikosch, T., Elementary Stochastic Calculus with Finance in View, World Scientific, 1999.

[21] Reiner, E., Convolution Methods for Exotic Options, presentation at Columbia University, March 2000.

[22] Takahashi, H. and Mori, M., Double exponential formulas for numerical integration, Publ. RIMS, Kyoto Univ., 9 (1974), 721-741. 